\title{
Relações Entre Apoio Social e Depressão Pós-Parto em Puérperas
}

\author{
Patrícia Alvarenga ${ }^{*}$,Emanuel M. Silva Palma, Luciana Maria Assis Silva \& Maria Virgínia Dazzani \\ Universidade Federal da Bahia, Salvador, Brasil
}

\begin{abstract}
RESUMO
Este estudo avalia a relação entre apoio social e depressão pós-parto em puérperas no primeiro mês de vida do bebê. Participaram 77 puérperas divididas em dois grupos. O grupo clínico foi composto por 39 mães com níveis de sintomatologia depressiva consideráveis segundo o Inventário Beck de Depressão (BDI). O grupo não-clínico foi formado por 38 mães com níveis mínimos segundo esse instrumento. As mães também responderam à Escala de Apoio Social do Medical Outcomes Study. Diferenças significativas foram encontradas entre os grupos em todos os fatores que compõem o instrumento de apoio social. As mães do grupo clínico obtiveram escores menores de apoio social quando comparadas às mães do grupo não-clínico, mostrando coerência com os achados da literatura.
\end{abstract}

Palavras-chave: depressão pós-parto; apoio social; puérperas.

\begin{abstract}
Associations Between Social Support and Postpartum Depression in Puerperal Mothers

This study assesses the relationship between social support and maternal depression in puerperal mothers in the first month postpartum. Participants were 77 puerperal mothers divided into two groups. The clinical group comprised 39 mothers with considerable levels of depressive symptomatology according to the Beck Depression Inventory (BDI). The non-clinical group comprised mothers with a minimum level according to the BDI. Mothers also completed the Medical Outcomes Study social support scale. Significant differences were found between the two groups on all factors of the MOS social support scale, with the clinical group scoring lower than the non-clinical group on all of them, showing consistency with other literature findings.
\end{abstract}

Keywords: postpartum depression; social support; puerperal mothers.

A depressão é considerada um dos transtornos mentais mais comuns entre a população e representa uma das queixas psiquiátricas mais identificadas em instituições de saúde mental (Kessler et al., 2003). Estudos da década de 90 já indicavam que a depressão seria a segunda principal causa de incapacidade no mundo em 2010 (Keller \& Bolland, 1998; Kessler, McGonagle, Swartz, Blazer, \& Nelson, 1993). A sua incidência é quase duas vezes maior em mulheres do que em homens e seu início ocorre normalmente entre 20 e 40 anos (Blazer, 2000). Dados epidemiológicos destacam que aproximadamente $15 \%$ das mulheres podem sofrer de depressão em qualquer momento de suas vidas, sendo que mães de bebês tendem a ser mais afetadas do que a população geral de mulheres
(Elgar, McGrath, Waschbusch, Stewart, \& Curtis, 2004).

Alguns estudos vêm tentando oferecer explicações para a discrepância na prevalência da depressão entre homens e mulheres. Embora exista alguma evidência que aponta para o papel de fatores biológicos e hormonais que tornariam as mulheres mais vulneráveis à depressão, mecanismos biológicos específicos relacionados ao gênero ainda não foram identificados (Nolen-Hoeksema \& Keita, 2003). Essa disparidade na prevalência de depressão entre os gêneros também tem sido atribuída às condições sociais. Estudos têm demonstrado que mulheres, especialmente mães, apresentam taxas mais elevadas de sintomas depressivos devido aos eventos estressores relacionados à criação

Endereço para correspondência: Patrícia Alvarenga - palvarenga66@gmail.com 
de filhos, à falta de uma rede de apoio social e ao baixo status socioeconômico (Rojas et al., 2010; Ruschi et al., 2007). Assim, o período pós-parto tem sido considerado um momento de elevada vulnerabilidade para o desenvolvimento de transtornos psiquiátricos em mulheres (O'Hara \& Swain, 1996).

O Diagnostic and Statistical Manual of Mental Disorders (DSM-IV-TR; American Psychiatric Association, 2000) não trata a depressão perinatal como uma categoria diagnóstica separada, e sim como um quadro de depressão maior. Esse diagnóstico, portanto, depende do preenchimento de uma série de critérios relacionados aos sintomas, duração e incapacidade definidos pelo DSM-IV-TR (2000). Entretanto, cabe ressaltar que o termo "pós-parto" aparece na categoria de transtornos de humor do DSM-IV-TR (2000), como um especificador, ou qualificador adicional, para se referir ao período de um episódio depressivo maior que se inicia dentro das quatro primeiras semanas após o nascimento do bebê.

Os estudos têm relatado uma prevalência da depressão pós-parto (DPP) que varia de 10 a $20 \%$ (O'Hara \& Swain, 1996), podendo em alguns casos chegar até a $40 \%$ (Coates, Schaefer, \& Alexander, 2004; Najman, Andersen, Bor, O’Callaghan, \& Williams, 2000). Em um estudo comparativo entre uma amostra brasileira e uma amostra britânica conduzido por Matijasevich et al. (2009), foi encontrada uma prevalência de depressão pós-parto de $16 \%$ da amostra de mães brasileiras e de $9,9 \%$ da amostra de mães britânicas 24 meses após o nascimento do bebê.

A depressão pós-parto tem início alguns dias ou semanas após o nascimento do bebê e caracteriza-se por sua duração relativamente mais longa em relação aos outros distúrbios de humor (por exemplo, síndrome de tristeza pós-parto) que podem afetar a mãe no puerpério (DSM-IV-TR, 2000). Seus sintomas podem incluir falta de apetite e de sono, dificuldade para dormir após amamentar o bebê, choro frequente, desatenção e falta de interesse por atividades antes consideradas agradáveis e ansiedade excessiva (Boyd, Le, \& Somberg, 2005). As mães podem também expressar sentimentos negativos ou ambivalentes em relação a seus bebês e ter dúvidas e preocupações quanto à sua habilidade de cuidar dos filhos, sendo também possível identificar ideação suicida em alguns casos. Se não for tratada, a depressão pós-parto pode afetar todos os membros da família. Paulson, Dauber e Leiferman (2006) conduziram um estudo recente em que puderam observar que $10 \%$ dos pais, cujas esposas apresentavam sintomas de depressão pós-parto, também apresentaram sintomas depressivos após o nascimento do bebê.

Por se tratar de uma condição de etiologia complexa, carente de explicações mais consistentes, a depressão pós-parto e sua elevada prevalência têm sido associadas a uma série de fatores biológicos, sociais e psicológicos, como as mudanças hormonais ocasionadas pela gestação (Marcus, 2009), um histórico prévio familiar ou individual de transtorno depressivo, o estado civil de solteira e a gravidez inesperada (Marcus, 2009; Matijasevich et al., 2009). Além desses fatores, alguns eventos estressores do ambiente doméstico ocasionados pelo baixo nível socioeconômico, os conflitos conjugais, a falta de uma rede de apoio social e as novas demandas resultantes dos cuidados com o recém-nascido (Beck, 2001; Najman et al., 2000) também tem estado relacionados a esse quadro.

Dentre as variáveis psicossociais, o apoio social tem sido considerado um dos mais fortes preditores de depressão pós-parto (Beck, 2001; Rojas et al., 2010), e a literatura tem se dedicado a definir, avaliar e identificar suas dimensões mais relevantes no estudo de sua associação com esse transtorno (Boutin-Foster \& Alexander, 2006; Bowling, 2003).

$\mathrm{O}$ apoio social foi definido amplamente como os recursos postos à disposição por outras pessoas (Bowling, 2003). Ele pode ser medido por meio da percepção individual do grau com que relações interpessoais correspondem a determinadas funções, como apoio afetivo, emocional e material (Boutin-Foster \& Alexander, 2006).

Um conceito importante no estudo de apoio social é o de rede social (social network) que foi definida como o grupo de pessoas com as quais os indivíduos mantêm contato ou alguma forma de vínculo social (Bowling, 2003) ou ainda como a rede de relacionamentos sociais da qual o indivíduo faz parte e as características dessas ligações (Berkman \& Glass, 2000). Para operacionalizar esse conceito têm sido utilizadas características como o número de pessoas com quem se mantém contato social (parentes, amigos e vizinhos), a frequência dessas interações, a composição da família, a condição de ter um cônjuge ou companheiro, a participação em atividades sociais em grupo, e ser membro e frequentar alguma religião ou organização de caráter voluntário (Berkman \& Glass, 2000; Bowling, 2003). A rede social pode ou não ofe- 
recer diferentes tipos de apoio em graus diversos. De acordo com Griep (2003), os efeitos benéficos da rede pessoal de apoio dependem da possibilidade de suprir os auxílios esperados pelo indivíduo. Dessa forma, a rede social pode ser definida como a estrutura social por meio da qual o apoio é fornecido.

De acordo com Boutin-Foster e Alexander (2006) e Bowling (2003), múltiplas dimensões funcionais do apoio social podem ser descritas. $\mathrm{O}$ apoio emocional está relacionado a receber demonstrações de afeto positivo e empatia e a ser encorajado a expressar sentimentos de empatia, carinho, amor, confiança, estima, afeto, escuta e interesse. $\mathrm{O}$ apoio afetivo refere-se a demonstrações físicas de afeto (por exemplo, abraços, beijos). A interação social positiva está relacionada a ter alguém com quem se distrair e fazer coisas agradáveis. O apoio de informação refere-se às informações que a pessoa recebe da rede, as quais poderá utilizar para lidar com problemas. Pode ser medido através da disponibilidade de aconselhamentos, sugestões, diretrizes e informações. Por fim, o apoio instrumental, ou material, é a disponibilidade de serviços práticos e recursos materiais, entre os quais estão incluídos, por exemplo, o auxilio nas tarefas da casa ou ajuda financeira em caso de necessidade emergencial.

De acordo com Chor, Griep, Lopes e Faerstein (2001), apesar de os mecanismos de ação exercidos pela rede e apoio social nos sistemas de defesa do organismo ainda não terem sido clarificados, duas hipóteses básicas são apresentadas. $\mathrm{Na}$ primeira, a rede e o apoio social atuariam "tamponando" a resposta do organismo em forma de doenças, que pode acontecer em consequência de grandes perdas ou rupturas emocionais (Cohen \& Pressman, 2004). O apoio social seria uma variável mediadora do efeito na relação entre o estresse e a doença. Os indivíduos que são expostos a estressores psicossociais e que simultaneamente sentem-se mais apoiados estariam mais protegidos dos efeitos nocivos dos estressores, do que os que se sentem menos apoiados. Na segunda hipótese, segundo Rodin (1986), o apoio social poderia reforçar a sensação de controle sobre a própria vida, o que implicaria em efeitos positivos sobre a saúde. $\mathrm{O}$ apoio social seria positivo para o indivíduo independentemente do nível de estresse. Os efeitos benéficos da participação e integração social são reconhecidos na sensação de bem estar psicológico, estando relacionados à autoestima, ao aumento da satisfação com a vida e à diminuição de sintomatologias como a ansiedade (Rodin, 1986).
Existem vários estudos relacionando à importância do apoio social sobre a saúde. De acordo Chor et al. (2001), um dos primeiros efeitos identificados da rede de apoio social sobre a saúde foi a forte e consistente associação inversa entre um índice multidimensional de laços sociais e a taxa geral de mortalidade (Berkman \& Syme, 1979). De acordo com Eng, Rimm, Fitzmaurice e Kawachi (2002), evidências apontam para uma relação inversa entre interação social e mortalidade. Os autores afirmam que, em geral, pessoas socialmente isoladas têm um risco aumentado de mortalidade em comparação com aquelas que mantêm relações com amigos, parentes e pessoas da comunidade.

Vários estudos demonstraram uma correlação significativa entre a percepção de apoio social e depressão durante a gravidez, revelando que mulheres grávidas que têm menos apoio social apresentam mais sintomas de depressão (Golbasi, Kelleci, Kisacik \& Cetin, 2010; Kazi et al., 2006; Marcus, Flynn, Blow, \& Barry, 2003; Records \& Rice, 2007; Rubertsson \& Waldenstrom, 2003). No estudo de Golbasi et al. (2010), a qualidade dos relacionamentos sociais esteve significativamente relacionada aos sintomas de depressão em mulheres. Segundo os autores, os profissionais de saúde precisam reconhecer que as mulheres com pouco apoio social podem estar em maior risco para a depressão pós-parto.

De acordo com Merchant, Affonso e Mayberry (1995), os sintomas depressivos, aos nove e 12 meses após o parto têm sido relacionados a um pobre relacionamento conjugal, o que sugere que relatos de insatisfação no casamento e a falta de apoio social são fatores de risco para a depressão pós-parto (Beck, 2001; Robertson, Grace, Wallington \& Stewart, 2004). Rojas et al. (2010) verificaram que a falta de apoio social e de uma relação estável associou-se de forma significativa com o aumento dos sintomas depressivos maternos. De acordo com Lee, Anderson, Horowitz e August (2009), o apoio social deve ser particularmente importante para pais de baixa renda, já que a prevalência de eventos estressores e depressão em mulheres pobres é praticamente duas vezes maior do que em mulheres com situação econômica mais favorável.

O estudo de Surkan, Peterson, Hughes e Gottlieb (2006) indicou que, de forma independente, tanto a rede social quanto o apoio social estavam inversamente relacionados com a sintomatologia depressiva. As mulheres que relataram ter dois ou mais amigos ou familiares disponíveis apresentaram menos sintomas 
depressivos. Essa relação já havia sido encontrada em estudos anteriores (Beck, 2001; Eberhard-Gran, Eskild, Tambs, Samuelsen, \& Opjordsmoen, 2002; Lee, Yip, Leung, \& Chung, 2004; Robertson et al., 2004; Merchant, Affonso, \& Mayberry, 1995). Os autores acreditam que as redes socias dão acesso às fontes de apoio, enquanto as medidas de apoio social avaliam a qualidade do apoio desses contatos da rede social. Em contraste com esses achados, Panzarine, Slater e Sharps (1995), num estudo com mães adolescentes, não encontraram diferenças significativas na frequência de relatos de recebimento de apoio social entre os grupos de mães não-deprimidas e de mães deprimidas.

Brugha et al. (1998) avaliaram a interação negativa com os parentes, amigos e outros contatos, como colegas de trabalho e conhecidos, e não encontraram relação significativa com sintomas depressivos. Contudo, o estudo de Surkan et al. (2006) indicou que as mulheres que relataram qualquer tipo de discriminação foram mais suscetíveis a desenvolver sintomas depressivos.A depressão pós-parto, além de afetar a vida da mãe, pode afetar o desenvolvimento da criança, predispondo-a a problemas emocionais e comportamentais (Elgar et al., 2004). A identificação de baixo apoio social durante a gravidez tem sido considerada um forte preditor de depressão pós-parto (Beck, 2001). Nesse contexto, o objetivo deste estudo foi avaliar a relação entre o apoio social percebido pela mãe e a depressão pós-parto no primeiro mês após o nascimento do bebê, comparando mães com sintomas de depressão e sem sintomas de depressão. A hipótese foi de que mães com sintomas depressivos teriam menores escores de apoio social.

\section{MÉTODO}

\section{Participantes}

Participaram do estudo 77 mães divididas em dois grupos. O grupo clínico foi composto por 39 mães que apresentaram níveis de depressão leve (12 a 19 pontos), moderado ( 20 a 35 pontos) ou grave (36 a 63 pontos), ou seja, que obtiveram um escore igual ou superior a 12 pontos no Inventário Beck de Depressão (BDI). O grupo não-clínico foi formado por 38 mães que apresentaram depressão mínima ou ausente, ou seja, que obtiveram um escore igual ou inferior a 11 pontos, no BDI.
As participantes foram selecionadas por conveniência em quatro maternidades públicas de Salvador, com base nos critérios descritos acima, dentre os participantes do estudo longitudinal Determinantes sociodemográficos, familiares e individuais do comportamento antissocial na infância (Alvarenga, Dazzani, Alfaya, Lordelo \& Piccinini, 2007). O objetivo desse estudo longitudinal é acompanhar famílias da gestação até o $11^{\circ}$ ano de vida das crianças, investigando uma série preditores e indicadores do comportamento antissocial na infância. Nesse estudo, todas as crianças eram do sexo masculino, um dos critérios de inclusão na amostra. De acordo com a literatura, as crianças do sexo masculino tendem a desenvolver mais problemas externalização e comportamento antissocial do que as crianças do sexo feminino (Colder, Mott, \& Berman, 2002). O estudo atende a todas as normas éticas de conduta em pesquisa com seres humanos, tendo sido aprovado pelo Comitê de Ética da Faculdade de Filosofia e Ciências Humanas da Universidade Federal da Bahia.

\section{Delineamento e procedimentos}

Foi utilizado um delineamento de grupos contrastantes (Nachmias \& Nachmias, 1996), sendo um grupo composto por mães que apresentaram níveis de sintomatologia depressiva leve (12 a 19 pontos), moderada (20 a 35 pontos) ou grave (36 a 63 pontos) no Inventário Beck de Depressão (BDI) e outro cujas mães apresentavam indicadores de depressão mínima (0 a 11 pontos) no mesmo instrumento. As gestantes foram contatadas e convidadas a participar do estudo longitudinal no terceiro trimestre da gestação em quatro maternidades públicas de Salvador, Bahia. No primeiro contato, as gestantes preencheram o Termo de Consentimento Livre e Esclarecido e a Ficha de Dados Sociodemográficos. As gestantes que preencheram os critérios do estudo longitudinal (estar esperando um bebê do sexo masculino, estar no $3^{\circ}$ trimestre de gestação e não apresentar complicações físicas graves durante a gravidez) foram contatadas posteriormente por telefone, para agendamento de uma visita domiciliar no primeiro mês de vida do bebê. Nessa ocasião, as mães responderam individualmente ao BDI (Beck \& Steer, 1993), ao Questionário sóciodemográfico e à Escala de Apoio Social do Medical Outcomes Study (Sherbourne \& Stewart, 1991). 


\section{Instrumentos}

Ficha de dados sociodemográficos e saúde da gestante: foi preenchida pelas gestantes na maternidade com o objetivo de selecionar possíveis participantes do estudo. Foram investigados alguns dados demográficos como a idade da gestante, escolaridade, profissão, estado civil, existência de outros filhos, estado de saúde durante a gestação e data prevista para o nascimento do bebê. $\mathrm{O}$ endereço e o telefone da gestante também foram solicitados para contato posterior.

Escala de Apoio Social do Medical Outcomes Study (Sherbourne \& Stewart, 1991): esta escala foi desenvolvida no Medical Outcomes Study (MOS) e mede a percepção dos indivíduos sobre o grau de apoio social. Foi utilizada a versão adaptada para a língua portuguesa (Chor et al., 2001; Faerstein, Lopes, Valente, Plá \& Ferreira, 1999; Griep, Chor, Faerstein, \& Lopes, 2003a; Griep, Chor, Faerstein \& Lopes, 2003b; Griep, Chor, Faerstein, Werneck, \& Lopes, 2005). O coeficiente alpha de Cronbach foi igual ou maior que 0,83 para todas as dimensões (Griep et al., 2005). A escala é constituída por 19 itens abrangendo cinco dimensões funcionais de apoio: material (quatro perguntas - provisão de recursos práticos e ajuda material); afetivo (três perguntas - demonstrações físicas de amor e afeto); emocional (quatro perguntas - expressões de afeto positivo, compreensão e sentimentos de confiança); interação social positiva (quatro perguntas - disponibilidade de pessoas para divertir-se ou relaxar) e informação (quatro perguntas - disponibilidade de pessoas para a obtenção de conselhos ou orientações). Cada item busca medir numa escala ordinal
(1 = "nunca", 2= "raramente", 3 = "às vezes", 4 = "quase sempre", 5 = "sempre") a frequência com que o sujeito acredita dispor de apoio social. Depois desse procedimento, as cinco dimensões foram avaliadas e reagrupadas por meio de análise fatorial em três fatores: apoio material, apoio afetivo/interação social positiva, emocional/informação. A pontuação nas 19 perguntas foi somada para gerar o escore total na escala.

Inventário Beck de Depressão - BDI (Beck \& Steer, 1993): este instrumento é uma escala sintomática de autorrelato, composta por 21 itens com diferentes alternativas de resposta sobre como o indivíduo tem se sentido recentemente, e que correspondem a níveis diferentes de gravidade da sintomatologia depressiva. A soma dos escores dos itens individuais fornece um escore total, que constitui um escore dimensional da intensidade da depressão, e pode ser classificado nos seguintes níveis: mínimo (0 a 11 pontos), leve (12 a 19 pontos), moderado (20 a 35 pontos) ou grave (36 a 63 pontos). Para o presente estudo, foi utilizada a versão em português do Inventário (Cunha, 2001; Cunha, Prieb, Goulart, \& Lemes, 1996), cuja consistência interna pelo alfa de Cronbach foi de 0,84 .

\section{RESULTADOS}

A Tabela 1 apresenta os dados para a depressão pós-parto do grupo clínico revelando o número de participantes em cada nível. A maioria das participantes $(59 \%)$ demonstra um nível sintomatologia leve enquanto que apenas $0,8 \%$ apresenta um escore de nível grave.

Tabela 1

Distribuição das Participantes do Grupo Clínico de Acordo Com o Nível de Sintomatologia Depressiva no BDI

\begin{tabular}{cl}
\hline Número de participantes & Nível de sintomatologia depressiva no BDI \\
$23(59 \%)$ & Leve (12 a 19 pontos) \\
$13(33 \%)$ & Moderado (20 a 35 pontos) \\
$03(0,8 \%)$ & Grave (36 a 63 pontos) \\
\hline
\end{tabular}

Com o objetivo de avaliar a equivalência entre os dois grupos, foram analisadas possíveis diferenças quanto às variáveis demográficas e fatores associados à gestação e à saúde das participantes .A Tabela 2 apresenta os dados demográficos das participantes do estudo. No grupo clínico e no grupo não-clínico, as idades das mães variaram entre 19 e 39 anos
$(M=28,57, D P=5,76 ; M=27,94, D P=5,23$ respectivamente). Houve uma diferença significativa entre os dois grupos quanto à escolaridade das mães $(t(68,25)=3,01 p=0,004)$, que foi maior no grupo não-clínico $(M=11,55, D P=1,78)$ do que no grupo clínico $(M=10,31, D P=2,37)$. Em relação à ocupação das mães, $14(35,9 \%)$ mães trabalhavam fora no 
grupo clínico, enquanto 14 (36,8\%) mães trabalhavam fora no grupo não-clínico. Em relação às variáveis relacionadas ao pai da criança não houve diferenças significativas. No grupo clínico, 32 pais $(82,1 \%)$ moravam com a criança, já no grupo não-clínico, 33 pais $(86,8 \%)$ residiam com a criança. A escolaridade do pai variou entre cinco e 14 anos $(M=10,26, D P=$ 4,96) no grupo clínico, e entre cinco e 16 anos $(M=$ $10,55, D P=2,9)$ no grupo não-clínico. No grupo clínico, $38(97,4 \%)$ pais trabalhavam fora, já no grupo não-clínico 33 pais $(89,5 \%)$ trabalhavam fora.
Em relação à variável número de irmãos da criança, no grupo clínico, a quantidade de irmãos variou entre zero e quatro $(M=0,74, D P=1,09)$, enquanto no grupo não-clínico, variou entre zero e três $(M=$ $0,76, D P=0,86)$. O número médio de moradores da casa foi $M=2,34(D P=1,55)$ no grupo clínico e $M=$ $2,27,(D P=1,58)$ no grupo não-clínico. Em relação aos moradores da casa, residiam apenas o casal e os filhos em $25(64,1 \%)$ famílias no grupo clínico e no grupo não-clínico em 25 (67,6\%) famílias.

Tabela 2

Características Demográficas das Participantes

\begin{tabular}{|c|c|c|c|c|}
\hline & $\begin{array}{c}\text { Grupo Clínico } \\
(\mathrm{n}=39)\end{array}$ & $\begin{array}{c}\text { Grupo Não-Clínico } \\
(\mathrm{n}=38)\end{array}$ & & \\
\hline \multicolumn{5}{|l|}{ Idade da gestante } \\
\hline M & 28,57 & 27,94 & $t=0,66^{*}$ & $p=0,51$ \\
\hline DP & 5,76 & 5,23 & & \\
\hline \multicolumn{5}{|l|}{ Escolaridade da mãe em anos } \\
\hline M & 10,31 & 11,55 & $t=3,01 * * *$ & $p=0,004$ \\
\hline DP & 2,37 & 1,78 & & \\
\hline \multicolumn{5}{|l|}{ Ocupação da mãe } \\
\hline Não trabalha fora & $25(64,1 \%)$ & $24(63,2 \%)$ & $X^{2}=0,007 * *$ & $p=0,93$ \\
\hline Trabalha fora & $14(35,9 \%)$ & $14(36,8 \%)$ & & \\
\hline \multicolumn{5}{|l|}{ Presença do pai } \\
\hline Reside com a criança & $32(82,1 \%)$ & $33(86,8 \%)$ & $X^{2}=0,755^{* *}$ & $p=0,39$ \\
\hline Não reside com a criança & $7(17,9 \%)$ & $5(13,2 \%)$ & & \\
\hline \multicolumn{5}{|l|}{ Escolaridade do pai em anos } \\
\hline M & 10,26 & 10,55 & $t=3,44 * * * *$ & $p=0,73$ \\
\hline DP & 4,96 & 2,90 & & \\
\hline \multicolumn{5}{|l|}{ Ocupação do pai } \\
\hline Não trabalha fora & $1(2,6 \%)$ & $4(10,5 \%)$ & $X^{2}=0,2 * *$ & $p=0,17$ \\
\hline Trabalha fora & $38(97,4 \%)$ & $34(89,5 \%)$ & & \\
\hline \multicolumn{5}{|l|}{ Renda Familiar } \\
\hline M & 820,03 & 861,73 & $t=0,56 * * * * *$ & $p=0,57$ \\
\hline DP & 534,17 & 524,57 & & \\
\hline \multicolumn{5}{|l|}{ Número de irmãos } \\
\hline M & 0,74 & 0,76 & $t=0,02 *$ & $p=0,97$ \\
\hline DP & 1,09 & 0,86 & & \\
\hline \multicolumn{5}{|l|}{ Número de moradores da casa } \\
\hline M & 2,34 & 2,27 & $t=0,09 *$ & $p=0,92$ \\
\hline DP & 1,55 & 1,58 & & \\
\hline \multicolumn{5}{|l|}{ Mora na residência } \\
\hline Casal ou casal e filhos & $25(64,1 \%)$ & $25(67,6 \%)$ & & \\
\hline Gestante e filhos & $3(7,7 \%)$ & $2(5,4 \%)$ & & \\
\hline Casal, pais e filhos & & $2(5,4 \%)$ & & \\
\hline Casal, filhos e outros parentes & $5(12,8 \%)$ & $2(5,4 \%)$ & $* * * * * *$ & \\
\hline Casal, filhos, pais e outros parentes & $1(2,6 \%)$ & $3(8,1 \%)$ & & \\
\hline Pais ou pais e outros parentes & $5(12,8 \%)$ & $3(8,1 \%)$ & & \\
\hline
\end{tabular}

Notas. ${ }^{*} g l=75 ;{ }^{* *} g l=1 ;{ }^{* * *} g l=68,25 ;{ }^{* * * *} g l=69 ;{ }^{* * * *} g l=72 ;{ }^{* * * * *}$ O teste qui-quadrado não pôde ser utilizado devido à baixa frequência esperada em algumas células. 
Com relação às condições da gestação e a saúde das gestantes, também não foram encontradas diferenças significativas. A Tabela 3 apresenta os dados sobre as condições da gestação nos dois grupos. No grupo clínico, $19(48,7 \%)$ mães estavam esperando o seu primeiro filho, no grupo não-clínico 15 (40,5\%) eram primíparas. Em relação ao planejamento da gravidez, no grupo clínico $15(38,5 \%)$ mães haviam pla- nejado a gravidez, enquanto no grupo não-clínico 17 $(44,7 \%)$ tinham feito o planejamento da gravidez. No grupo clínico, $10(25, \%)$ mães tiveram complicações na gravidez, já no grupo não-clínico, sete $(18,4 \%)$ mães tiveram complicações. Em relação a problemas de saúde, quatro $(10,3 \%)$ mães do grupo clínico tinham problemas de saúde, enquanto no grupo nãoclínico sete $(18,4 \%)$ apresentavam problemas de saúde.

Tabela 3

Condições da Gestação nos Grupos Clínico e Não-clínico

\begin{tabular}{|c|c|c|c|c|}
\hline & $\begin{array}{l}\text { Grupo Clínico } \\
(\mathrm{n}=39)\end{array}$ & $\begin{array}{l}\text { Grupo Não-clínico } \\
\qquad(\mathrm{n}=38)\end{array}$ & & \\
\hline \multicolumn{5}{|l|}{ Primaparidade } \\
\hline Primípara & $19(48,7 \%)$ & $15(40,5 \%)$ & \multirow[t]{2}{*}{$X^{2}=0,51$} & \multirow[t]{2}{*}{$p=0,47$} \\
\hline Multípara & $20(51,3 \%)$ & $23(59,5 \%)$ & & \\
\hline \multicolumn{5}{|c|}{ Planejamento da gravidez } \\
\hline Esperada & $15(38,5 \%)$ & $17(44,7 \%)$ & \multirow[t]{2}{*}{$X^{2}=0,31$} & \multirow[t]{2}{*}{$p=0,57$} \\
\hline Não esperada & $24(61,5 \%)$ & $21(55,3 \%)$ & & \\
\hline \multicolumn{5}{|c|}{ Complicação na gravidez } \\
\hline Presença & $10(25,6 \%)$ & $7(18,4 \%)$ & \multirow[t]{2}{*}{$X^{2}=0,58$} & \multirow[t]{2}{*}{$p=0,44$} \\
\hline Ausência & $29(74,4 \%)$ & $31(81,6 \%)$ & & \\
\hline \multicolumn{5}{|l|}{ Problemas de saúde } \\
\hline Presença & $4(10,3 \%)$ & $7(18,4 \%)$ & \multirow[t]{2}{*}{$X^{2}=0,34$} & \multirow[t]{2}{*}{$p=0,24$} \\
\hline Ausência & $35(89,7 \%)$ & $31(81,6 \%)$ & & \\
\hline
\end{tabular}

Nota. $g l=1$.

Por fim, foram comparados os escores médios dos dois grupos nos três fatores que compõem a Escala de Apoio Social do MOS: afetivo ou interação social, emocional ou informação e material. Para verificar possíveis diferenças nos fatores que compõem o apoio social nos dois grupos, foi utilizada a análise de covariância (ANCOVA) com a inclusão da variável escolaridade materna como covariantes. O teste $t$ de Student foi utilizado para comparar os escores obtidos na Escala de Apoio Social de MOS nos grupos clínico e não-clínico. A Tabela 4 descreve as médias, desvios- padrões, valor $F$ e nível de significância no escore total, e nos três fatores que compõem o apoio social ( apoio afetivo/interação social positiva, apoio emocional/informação e apoio material) nos grupos clínico e não-clínico. Foram encontradas diferenças significativas entre os dois grupos em todos os fatores da Escala de Apoio Social, sendo que o grupo clínico, composto por mães com sintomatologia depressiva leve, moderada ou grave, apresentou escores significativamente menores em todos os fatores se comparadas às mães do grupo não-clínico.

Tabela 4

Média, Desvio-Padrão, Valor F e Nível de Significância dos Escores na Escala de Apoio Social nos Grupos Clínico e Não-clínico

\begin{tabular}{lcccc}
\hline & Grupo Clínico & Grupo Não-Clínico & $F$ & $p$ \\
& $M(D P)$ & $M(D P)$ & & \\
Fator apoio afetivo / interação social positiva & $21,53(5,33)$ & $24,72(3,42)$ & 6,63 & 0,012 \\
Fator apoio emocional / informação & $22,71(5,63)$ & $25,29(5,14)$ & 4,49 & 0,04 \\
Fator apoio material & $10,79(2,08)$ & $12,89(3,26)$ & 4,41 & 0,04 \\
Escore total & $54,56(13,59)$ & $62,34(9,42)$ & 7,46 & 0,008 \\
\hline
\end{tabular}

Nota. $g l=1$ 


\section{DISCUSSÃO}

O objetivo do presente estudo foi avaliar a relação entre o apoio social e a depressão pós-parto em puérperas no primeiro mês após o nascimento do bebê. Os resultados encontrados apoiaram a hipótese inicial de que as mães com sintomas depressivos apresentariam menores escores de apoio social, demonstrando que o grupo clínico, composto por mães com sintomatologia depressiva, apresentou escores significativamente menores em todos os três fatores e no escore total da escala de apoio social, quando comparado ao grupo não-clínico. Esses resultados são consistentes com os achados do estudo conduzido por Xie, He, Koszicki, Walker e Wen (2009) com uma amostra de 534 mães chinesas. Esses autores verificaram que o baixo índice ou falta de apoio social nos períodos pré e pós-parto estava associado a um maior risco de depressão pósparto, sendo que a dimensão apoio objetivo (grau de apoio prático que a rede social é capaz de fornecer, como cuidar do bebê) foi a mais fortemente associada aos sintomas depressivos maternos no pós-parto.

Horwitz, Brigss-Gowan, Stoffer-Isser e Carter (2007) descobriram que mães com baixo apoio social relataram mais sintomas depressivos nos primeiros meses após o parto. Nessa mesma perspectiva, Ritter et al. (2000) demonstraram que uma maior satisfação com o apoio social estava relacionada a um nível reduzido de depressão pós-parto. Esses achados são coerentes com os resultados do presente estudo e demonstram a importância de investigar a quantidade, a qualidade e a satisfação das mães com o apoio social que recebem. Outros estudos também dão suporte à hipótese de que altos níveis de apoio social associam-se de forma significativa com níveis menores de depressão pós-parto (Cairney, Boyle, Offord, \& Racine, 2003; Surkan et al., 2006).

É provável que no período pós-parto, devido às novas demandas impostas pelos cuidados com o bebê, as mães se sintam menos disponíveis emocionalmente e apresentem mais sintomas depressivos Além do mais, outros fatores como características temperamentais do bebê (por exemplo, bebês de temperamento difícil), baixo status socioeconômico, baixo grau de instrução da mãe, falta de um parceiro para auxiliar nas atividades, entre outros, podem contribuir para o surgimento ou agravamento dos sintomas depressivos maternos (Rojas et al., 2010; Ruschi et al., 2007). Nesse contexto, uma rede social consistente, capaz de oferecer ajuda e satisfazer algumas necessidades da nova mãe, como por exemplo, cuidar do bebê por algum tempo ou fornecer informações úteis sobre os cuidados com o bebê, pode atenuar os efeitos deletérios da depressão pós-parto, tanto sobre a saúde da mãe, quanto sobre a qualidade de sua interação com o filho.

De forma semelhante ao presente estudo, outro estudo brasileiro conduzido por Fonseca, Silva e Otta (2010) com 261 mães descobriu correlações negativas estatisticamente significativas entre os escores de depressão pós-parto e apoio social recebido durante a gravidez nos fatores emocional/informação e afetivo/interação positiva. Para as autoras, esses resultados sugerem que o apoio social durante a gestação pode funcionar como um fator de proteção para os sintomas depressivos, ou seja, quanto mais apoio social a mãe receber, menos intensos serão seus sintomas depressivos no pós-parto. Nessa mesma perspectiva, Webster, Nicholas, Velacott, Cridland e Fawcett (2011), ao investigar a qualidade de vida, a depressão pós-parto e o impacto do apoio social em uma amostra de 320 mulheres australianas, descobriram que mulheres que relataram ter baixo apoio social foram mais vulneráveis a apresentação de indicadores de depressão após o nascimento do bebê.

Ao averiguar na literatura, os mecanismos que explicam as dificuldades interpessoais de pessoas deprimidas e o possível impacto desses déficits sobre sua rede de apoio social, Hammen, Shih e Brennan (2004) verificaram que pessoas deprimidas tendem a induzir humor negativo nos outros, demonstram mais carência e procuram reasseguramento com mais frequência, além de não terem um bom repertório de habilidades sociais. Esses aspectos contribuem para que elas provoquem reações negativas e afastamento em seu entorno social. Para Segrin e Rynes (2009), isso acontece porque as pessoas deprimidas violam certas regras de comunicação como a responsividade, a cortesia e as expectativas de envolvimento, o que contribui para interações mais aversivas e pouco gratificantes com os outros. No contexto da depressão pósparto e do apoio social, analisado sob a perspectiva dos modelos interpessoais de depressão, é possível então destacar que não só a falta de uma rede social pode contribuir para a depressão pós-parto, mas também que o próprio estilo de interação da mãe deprimida (por ex.: queixas excessivas, solicitação de reasseguramento frequente, apatia, etc.) pode contribuir para o afastamento das pessoas que compõem a sua rede. A 
complexidade dessas relações e suas possíveis implicações para o tratamento da depressão pós-parto merecem mais atenção dos pesquisadores.

Embora os resultados do presente estudo sejam coerentes com outros reportados na literatura, algumas limitações também devem ser levadas em consideração. Em primeiro lugar, o número reduzido de participantes neste estudo, bem como algumas de suas características como status econômico baixo e a diferença de grau de instrução, medida em anos de escolaridade, entre o grupo clínico e o não clínico, representam importantes particularidades da amostra. Além disso, os pesquisadores têm adotado instrumentos diferentes para avaliar tanto a depressão pós-parto quanto o apoio social. Consequentemente, os pontos de cortes adotados, bem como as dimensões avaliadas, são distintos, o que impede comparações mais consistentes entre esses estudos.

Apesar dessas limitações, os resultados deste estudo reforçam a noção de que o apoio social é um fator importante para a compreensão do fenômeno da depressão pós-parto. Desse modo, a avaliação das redes de apoio social da gestante no período pré-natal pode fornecer subsídios relevantes para intervenções psicossociais que visem a fortalecer o apoio social recebido por essas mães, minimizando, dessa forma, sua vulnerabilidade aos sintomas depressivos. Boyd, Diamond e Bourjoully (2006) incluíram com relativo sucesso o apoio social entre os componentes de seu programa de auxílio a mães deprimidas. Nesse sentido, recomenda-se que futuras investigações explorem o potencial desse foco de intervenção no acompanhamento de gestantes nos centros de saúde, e sua repercussão em fatores como a saúde mental da puérpera e a relação mãe-bebê.

\section{REFERÊNCIAS}

Alvarenga, P., Dazzani, M. V., Alfaya, C. A., Lordelo, E., \& Piccinini, C. (2007). Determinantes sociodemográficos, familiares e individuais do comportamento antissocial na infância. Manuscrito não publicado.

American Psychiatric Association. (2000). Diagnostic and statistical manual of mental disorders (4th ed., text rev.). Washington, DC: Author.

Beck, A. T., \& Steer, R. A. (1993). Beck Depression Inventory Manual. San Antonio, TX: Psychological Corporation.

Beck. C. T. (2001). Predictors of postpartum depression: An update. Nursing Research, 50, 275-285.
Berkman, L. F., \& Glass, T. (2000). Social integration, social networks, social support and health. In L. F. Berkman \& I. Kawachi (Eds.), Social Epidemiology (pp. 137-173). New York, NY: Oxford University Press.

Berkman, L. F., \& Syme, S. L. (1979). Social networks, host resistance, and mortality: A nine-year follow-up study of Alameda County residents. American Journal of Epidemiology, 109, 186-204.

Blazer, D. G. (2000). Mood disorders: Epidemiology. In B. J. Sadock \& V. A. Sadock (Eds.), Kaplan and Sadock's comprehensive textbook of psychiatry $\left(7^{\text {th }}\right.$ edition, vol. 1, pp. 12981308). Philadelphia, PA: Lippincott, Williams \& Wilkins.

Boutin-Foster, C., \& Alexander, J. (2006). Development and validation of the tangible, informational, and emotional support survey. Journal of Cardiopulmonary Rehabilitation, 26, 307313.

Bowling, A. (2003). Measuring social networks and social support: $2^{\text {nd }}$ edition. In A. Bowling (Ed.), Measuring health: A review of quality of life measurement scales (pp. 91-109). Buckingham, England: Open University Press.

Boyd, R. C., Diamond, G. S., \& Bourjolly, J. N. (2006). Developing a family-based depression prevention program in urban community mental health clinics: A qualitative investigation. Family Process, 45, 187-203.

Boyd, R. C., Le, H. N., \& Somberg, R. (2005). Reviewing screening instruments for postpartum depression. Archives of Women's Mental Health, 9, 21-25.

Brugha, T. S., Sharp, H. M., Cooper, S. A., Weisender, C., Britto, D., Shinkwin, R., Sherrif, T., \& Kirwan, P. H. (1998). The Leicester 500 Project: Social support and the development of postnatal depressive symptoms, a prospective cohort survey. Psychological Medicine, 28, 63-79.

Cairney, J., Boyle, M., Offord, D. R., \& Racine, Y. (2003). Stress, social support and depression in single and married mothers. Social Psychiatry and Psychiatric Epidemiology, 38, 442-449.

Chor, D., Griep, R. H., Lopes, C. S., \& Faerstein, E. (2001). Medidas de rede e apoio social no Estudo Pró-Saúde: Pré-testes e estudo piloto. Cadernos de Saúde Pública, 17, 887-896.

Coates, A. O., Schaefer, C. A., \& Alexander, J. L. (2004). Detection of postpartumdepression and anxiety in a large health plan. Journal of Behavioral Health Services and Research, 31, 117133.

Cohen, S., \& Pressman, S. (2004). The stress-buffering hypothesis. In N. Anderson (Ed.), Encyclopedia of health and behavior (pp. 696-698). Thousand Oaks, CA: Sage Publications.

Colder, C. R., Mott, J. A., \& Berman, A. S. (2002). The interactive effects of infant activity level and fear on growth trajectories of early child behavior problems. Developmental and Psychopathology, 14, 1-23.

Cunha, J. A. (2001). Escalas Beck. São Paulo: Casa do Psicólogo.

Cunha, J. A., Prieb, R. G. G., Touginha, L. A., \& Goulart, P. M. (1996). Depressão em universitários da PUCRS. Psico, 27, $97-$ 109.

Eberhard-Gran, M., Eskild, A., Tambs, K., Samuelsen, S. O. \& Opjordsmoen, S. (2002). Depression in postpartum and nonpostpartum women: Prevalence and risk factors. Acta Psychiatrica Scandinavica, 106, 426-433. 
Elgar, F. J., McGrath, P. J., Waschbusch, D. A., Stewart, S. H., \& Curtis, L. J. (2004). Mutual influences on maternal depression and child adjustment problems. Clinical Psychology Review, 24, 441-459.

Eng, M. P., Rimm, B. E., Fitzmaurice, G., \& Kawachi, I. (2002). Social ties and change in social ties to subsequent total and cause-specific mortality in coronary heart: Heart disease incidence in men. American Journal of Epidemiology, 155, 700709.

Faerstein, E., Lopes, C. S., Valente, K., Plá, M. A. S., \& Ferreira, M. B. (1999). Pré-testes de um questionário multidimensional autopreenchível: A experiência do Estudo Pró-Saúde. PHYSIS - Revista de Saúde Coletiva, 9, 117-130.

Fonseca, V. R. J. R. M., Silva, G. A., \& Otta, E. (2010). Relação entre depressão pós-parto e disponibilidade emocional materna. Caderno de Saúde Pública, 26, 738-746.

Golbasi, Z., Kelleci, M., Kisacik, G., \& Cetin, A. (2010). Prevalence and correlates of depression in pregnancy among turkish women. Maternal and Child Health Journal, 14, 485-491.

Griep, R. H. (2003). Confiabilidade e validade de instrumentos de medida de rede social e apoio social utilizados no Estudo Pró-Saúde (Tese de doutorado). Escola Nacional de Saúde Pública, Fundação Oswaldo Cruz, Rio de Janeiro.

Griep, R. H., Chor, D., Faerstein, E., \& Lopes, C. (2003a). Apoio social: Confiabilidade teste-reteste de escala no Estudo Pró-Saúde. Cadernos de Saúde Pública, 19, 625-634.

Griep, R. H., Chor, D., Faerstein, E., \& Lopes, C. (2003b). Confiabilidade teste-reteste de aspectos da rede social no Estudo Pró-Saúde. Revista de Saúde Pública, 37, 379-385.

Griep, R. H., Chor, D., Faerstein, E., Werneck, G. L., \& Lopes, C. (2005). Validade de constructo de escala de apoio social do Medical Outcomes Study adaptada para o português no Estudo Pró-Saúde. Cadernos de Saúde Pública, 21, 703-714.

Hammen, C., Shih, J., \& Brennan, P. (2004). Intergenerational transmission of depression: Test of an interpersonal stress model in a community sample. Journal of Consulting and Clinical Psychology, 72, 511-522.

Horwitz, S. M., Brigss-Gowan, M. J., Storfer-Isser, A., \& Carter, A. S. (2007). Prevalence, correlates, and persistence of maternal depression. Journal of Women's Health, 16, 678-691.

Kazi, A., Fatmi, Z., Hatcher, J., Kadir, M. M., Niaz, U., Gail, A., (2006). Social environment and depression among pregnant women in urban areas of Pakistan: Importance of social relations. Social Science and Medicine, 63, 1466-1476.

Keller, M. B., \& Boland, R. J. (1998). Implications of failing to achieve successful long-term maintance treatment of recurrent unipolar depression. Biological Psychiatry, 44, 348-360.

Kessler, R. C., McGonagle, K. A., Swartz, M., Blazer, D. G., \& Nelson, C. B. (1993). Sex and depression in the national comorbidity survey I: Lifetime prevalence, chronicity and recurrence. Journal of Affective Disorders, 29, 85-96.

Kessler, R. C., Berglund, P., Demler, O., Jin, R., Koretz, D., Merikangas, K. R., Rush, J., Walters, E. E., \& Wang, P. S. (2003). The epidemiology of major depressive disorder: Results from the National Comorbidity Replication. Journal of the American Medical Association, 289, 3095-3105.
Lee, C. S., Anderson, J. R., Horowitz, J. L., \& August, G. J. (2009). Family income and parenting: The role of parental depression and social support. Family Relations, 58, 417-430.

Lee, D. T., Yip, A. S., Leung, T. Y., \& Chung, T. K. (2004). Ethnoepidemiology of postnatal depression: Prospective multivariate study of sociocultural risk factors in a Chinese population in Hong Kong. British Journal of Psychiatry, 184, 34-40.

Marcus, S. (2009). Depression during pregnancy: Rates, risks, and consequences. The Canadian Journal of Clinical Pharmacology, 16, 15-22.

Marcus, S. M., Flynn, H. A., Blow, F. C., \& Barry, K. L . (2003). Depressive symptoms among pregnant women screened in obstetric settings. Journal of Women's Health, 12, 373-380.

Matijasevich, A., Golding, J., Smith, G. D., Santos, I. S., Barros, A., \& Victora, C. (2009). Differentials and income-related inequalities in maternal depression during the first two years after childbirth: Birth cohort studies from Brazil and the UK. Clinical Practice and Epidemiology in Mental Health, 5. doi: 10.1186/1745-0179-5-12

Merchant, D. C., Affonso, D. D., \& Mayberry, L. J. (1995) Influence of marital relationship and child-care stress on maternal depression symptoms in the postpartum. Journal of Psychosomatic Obstetrics and Gynaecology, 16, 193-200.

Nachmias, C., \& Nachmias, D. (1996). Research methods in the social sciences. London, UK: Arnolds.

Najman, J. M., Andersen, M. J., Bor, W., O'Callaghan, M. J., Williams, G. M. (2000). Postnatal depression: Myth or reality Maternal depression before and after the birth of a child. Social Psychiatry and Psychiatric Epidemiology, 35, 19-27.

Nolen-Hoeksema, S., Keita, G. P. (2003). Women and depression: Introduction. Psychology of Women Quarterly, 27, 89-90

O'Ilara, M. W., \& Swain, A. M. (1996). Rates and risk of postpartum depression: A meta-analysis. International Review of Psychiatry, 8, 37-54.

Panzarine, S., Slater, E., \& Sharps, P. (1995). Coping, social support, and depressive symptoms in adolescent mothers. Journal of Adolescent Health, 17, 113-119.

Paulson, J. F., Dauber, S., \& Leiferman, J. A. (2006). Individual and combined effects of postpartum depression in mothers and fathers on parenting behavior. Pediatrics, 118, 659-668.

Records, K., \& Rice, M. (2007). Psychosocial correlates of depression symptoms during the third trimester of pregnancy. Journal of Obstetric, Gynecologic and Neonatal Nursing, 36, 231-242.

Ritter, C., Hobfoll, S. E., Lavin, J., Cameron, R. P., \& Hulsizer, M. R. (2000). Stress, psychological resources, and depressive symptomatology during pregnancy in low-income, inner-city women. Health Psychology, 19, 576-585.

Robertson, E., Grace, S., Wallington, T., \& Stewart, D. E. (2004). Antenatal risk factors for postpartum depression: A synthesis of recent literature. General Hospital Psychiatry, 26, 289-295.

Rodin, J. (1986). Aging and health: Effects of the sense of control. Science, 233, 1271-1276.

Rojas, G., Fritsch, R., Guajardo, V., Rojas, F., Barroilhet, S., \& Jadresic, E. (2010). Caracterización de madres deprimidas em el posparto. Revista Médica de Chile, 138, 536-542. 
Rubertsson, C., \& Waldenstrom, U. (2003). Depressive mood in early pregnancy: Prevalence and women at risk in a national Swedish sample. Society for Reproductive and Infant Psychology, 21, 113-121.

Ruschi, G. E. C., Sun, S. Y., Mattar, R., Filho, A. C., Zandonades, E., \& Lima, V. J. (2007). Aspectos epidemiológicos da depressão pós-parto em amostra brasileira. Revista de Psiquiatria do Rio Grande do Sul, 29, 274-280.

Segrin, C., \& Rynes, K. N. (2009). The mediating role of positive relations with others in associations between depression, social skills, and perceived stress. Journal of Research in Personality, $43,962-971$

Sherbourne, C. D., \& Stewart, A. L. (1991). The MOS social support survey. Social Science Medicine, 38, 705-714.

Surkan, P. J., Peterson, K. E., Hughes, M. D., \& Gottlieb, B. R. (2006). The role of social networks and support in postpartum women's depression: A multiethnic urban sample. Maternal and Child Health Journal, 10, 375-383.

Webster, J., Nicholas, C., Velacott, C., Cridland, N., \& Fawcett, L. (2011). Quality of life and depression following childbirth: Impact of social support. Midwifery, 27, 245-274.

Xie, R. H., He, G., Koszycki, D., Walker, M., \& Wen, S. W. (2009). Prenatal social support, postnatal social support, and postpartum depression. Annals of Epidemiology, 19, 637-43.

Recebido: 09/12/201

Última revisão: 10/10/2012 Aceito: 16/12/2012 
